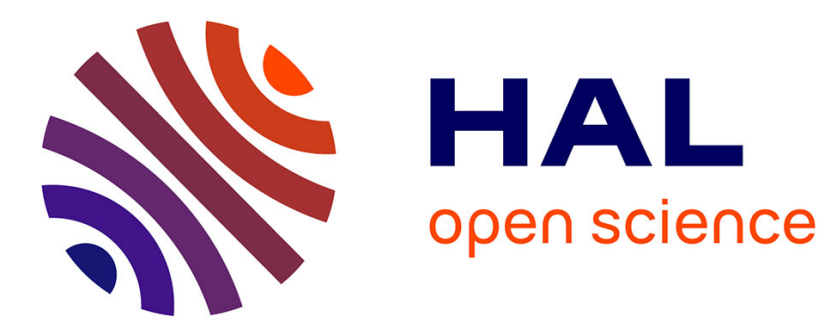

\title{
The strategy structure of some coalition formation games
}

Gabrielle Demange

\section{To cite this version:}

Gabrielle Demange. The strategy structure of some coalition formation games. 2006. halshs00590290

\section{HAL Id: halshs-00590290 \\ https://shs.hal.science/halshs-00590290}

Preprint submitted on 3 May 2011

HAL is a multi-disciplinary open access archive for the deposit and dissemination of scientific research documents, whether they are published or not. The documents may come from teaching and research institutions in France or abroad, or from public or private research centers.
L'archive ouverte pluridisciplinaire HAL, est destinée au dépôt et à la diffusion de documents scientifiques de niveau recherche, publiés ou non, émanant des établissements d'enseignement et de recherche français ou étrangers, des laboratoires publics ou privés. 


\title{
Paris-Jourdan Sciences Economiques \\ 48, BD JOURDAN - E.N.S. - 75014 PARIS \\ TEL. : 33(0) 143136300 - FAX : 33 (0) 143136310 \\ www.pse.ens.fr
}

WORKING PAPER Nº 2006 - 38

\author{
The strategy structure \\ of some coalition formation games
}

Gabrielle Demange

JEL Codes : C71, D7

Keywords : coalition formation, assignment, manipulability, substitutes, incremental value, Vickrey-Clarke-Groves mechanism. 


\title{
The strategy structure of some coalition formation games
}

\author{
Gabrielle DEMANGE*
}

December 8, 2006

\begin{abstract}
In coalitional games with side payments, the core predicts which coalitions form and how benefits are shared. The predictions however run into difficulties if the core is empty or if some coalitions benefit from not blocking truthfully. These difficulties are analyzed in games in which an a priori given collection of coalitions can form, as the collection of pairs of buyer-seller in an assignment game. The incentive properties of the core and of its selections are investigated in function of the collection. Furthermore the relationships with Vickrey-Clarke-Groves mechanisms are drawn.
\end{abstract}

Keywords : coalition formation, assignment, manipulability, substitutes, incremental value, VickreyClarke-Groves mechanism.

JEL Classification : C71, D7

*EHESS at PSE 48 bd Jourdan, 75014 Paris, France. e-mail demange@pse.ens.fr. I thank an anonymous referee and Pablo Amoros for excellent remarks and suggestions. 


\section{Introduction}

The idea of using the core as a model for assessing the stability of arrangements made within a society has been proved quite fruitful in various contexts. In particular, the core is useful in the situations that call for the society to split up into smaller self-sufficient groups, provided there are no externalities across these groups. In these situations, the threat of blocking puts constraints not only on how benefits are shared but also on which coalitions form. Shapley and Shubik (1971) were among the first to use this insight in their analysis of the "assignment game". In a two-sided market in which only pairs of buyer and seller are worth forming, possible outcomes specify which pairs end up making a transaction and at what price. Stable outcomes always exist, supported by equilibrium prices.

The action of blocking is the driving force underlying the concept of the core. The predictions given by the core may run into difficulties if some individuals or coalitions benefit from not blocking "truthfully". To define truthful blocking independently of any specific coalition formation process, a solution is to consider the one-shot process that assigns the core to each possible configuration of individual preferences and to study its manipulability. Since the core is not in general a singleton, several definitions of manipulability are possible. ${ }^{1}$ For our purpose, the appropriate notion is the manipulability by "optimistic" individuals or coalitions as introduced in an earlier paper (Demange 1987). Consider an individual who belongs to all coalitions blocking a given alternative. It may happen that he prefers that alternative to each alternative in the core. If he indeed expects an outcome in the core to obtain, why should he agree to block? As explained in the paper, no such objection can be raised if the core correspondence is not manipulable by an optimistic individual. Extending similar considerations to coalitions leads to the concept of manipulability by optimistic coalitions.

Although quite surprising at first sight, the non manipulability of the core is not an innocuous condition (we omit 'optimistic' from now). For example the core of an exchange economy can be manipulable. Instead, the core of an assignment game is not manipulable by the essential coalitions, that is neither by optimistic individuals nor by pairs of buyer-seller. This paper analyzes more general coalitional games where, as in an assignment game, there is an a priori given collection of admissible coalitions. Because of lack of communication or institutional reasons or organizational constraints for instance, coalitions outside the collection are unable to form. Our first objective is to determine which collections allow for the core to be non manipulable.

The analysis is conducted in the following setting. A finite number of individuals may organize themselves into pairwise disjoint admissible coalitions (staying single is always admissible). Players only care about the coalition they join and the amount of money they receive (or give), and utility is transferable. Thus, an outcome specifies how the society splits up into admissible coalitions and which transfers each coalition will implement within its members. As is well known, the core may be empty, that is no stable outcome exists. Our first result states that both difficulties -emptiness and manipulability of the core- are solved simultaneously. More precisely, consider a collection of

\footnotetext{
${ }^{1}$ These definitions depend on how preferences over outcomes are extended to preferences over subsets of outcomes. Manipulability by an optimistic individual is obtained when a subset is evaluated through its most preferred element(s) (see section 3).
} 
admissible coalitions for which a core outcome exists whatever the players preferences, as introduced by Kaneko and Wooders (1982). Given such a collection, the (nonempty) core is non manipulable in the optimistic sense by admissible coalitions (Theorem 1). Furthermore, the result is related with Vickrey-Clarkes-Groves mechanisms (Vickrey 1961, Clarke 1971, and Groves 1973) : it holds because an admissible coalition can achieve its incremental value over the remaining players at a core outcome. ${ }^{2}$ Aside assignment games, interesting examples are given by tree-hierarchical structures in which only connected coalitions can be admissible (see Demange 2004 for a detailed analysis).

To address further the relevance of the core, the second objective of the paper is to analyze the incentive properties of core selections. The first result is negative. No selection is strategy-proof for all players. Even worse, manipulation is pervasive: an individual can manipulate whenever the selected outcome is not one of his preferred core outcomes. A selection however may be strategy-proof for a subset of players. As is well known in the assignment game, there is a generalization of Vickrey's single object auction that gives the incentives to all buyers to reveal their true valuations (Leonard 1983, Demange et al 1986). It turns out that a simple property, the absence of a chain between players, characterizes the collections of admissible coalitions that allow for a selection to be strategy-proof for these players (Theorem 2). These collections ensure that the players are substitutes in the sense of Shapley and Shubik (1971) independently of their preferences. In tree-hierarchical games for instance, two players are substitutes only if no connected coalition containing both players is admissible.

The manipulability of core outcomes has been investigated in similar settings without money, starting with marriage problems by Dubins and Freedman (1981), and housing markets by Roth and Postlewaite (1977). Excluding side payments makes the analysis rather different. An outcome is simply specified by a partition of the society. The core, necessarily discrete, is single valued for some collections of admissible coalitions. Single valued cores allow the standard concept of strategyproofness to be used (Papai 2004). Also, in a generalized matching market, the existence of a strategyproof rule with desirable properties is closely related to single-valued cores (Sönmez 1999). With side payments, a core is almost never single valued so that similar results are hopeless.

Stability and incentives issues are also the subject of a vast literature in another type of extension of the assignment game. Keeping a two sided structure, buyers (firms) may want to buy several objects (workers), as in a job market (Crawford and Knoer 1981) or in various allocation problems such as spectrum bandwidth auctions (Cramton 1995). The existence of stable outcomes, the design of auction mechanisms to reach them, and the relationships with Vickrey payoffs have been studied (see e.g. Ausubel 2004). In particular, as in this paper, Bikhchandani and Ostroy $(2002,2006)$ show the importance of the property of substitutes players and use linear programming technics. In contrast however, restrictions on preferences, not on coalitions, are the keys to restore stable outcomes or to state various properties.

Finally, our concern on justifying the blocking condition is related with the literature that recog-

\footnotetext{
${ }^{2}$ The importance of the notion of incremental value, also called marginal value or marginal product or (for a single individual) Vickrey payoff, has been recognized in various contexts. Shapley and Shubik (1971) used it to introduce the notion of substitute players. For a thorough analysis of the relationships between incremental values and competitive behavior, see Makowski and Ostroy (1987).
} 
nizes that blocking should not been taken as granted when coalitions look ahead and try to figure out the ultimate consequences of their moves. Based on this observation, various behaviors can be justified so that only consistent sets of outcomes are predicted, as explained by Chwe (1994) in a general context. For an application to coalition formation possibly with spill-overs across coalitions, see for example Barbera and Gerber (2003) and Diamantoudi and Xue (2003).

The paper is organized as follows. Section 2 introduces the coalitional formation model, illustrates it with some examples, and defines incremental values and stable payoffs. Section 3 discusses various definitions of manipulability for the core correspondence and provides a justification for the manipulability concept by optimistic coalitions. The non manipulability result on core correspondences is given in Section 4. Section 5 is devoted to strategy-proof selections of the core, and the final section presents conclusions.

\section{The model and examples}

It is worth recalling the basic features and properties of the two-sided market introduced by Shapley and Shubik (1971). In the market, say a real estate market, there are two types of agents, prospective purchasers, the "buyers" and homeowners, the "sellers". Each buyer is interested in buying only one house. The $i$-th seller values his own house at $c_{i}$ dollars while the $j$-th buyer values the same house at $h_{i j}$ dollars. The important data are the "essential" coalitions, the pairs of buyer-seller, and the total value they derive by forming, here $h_{i, j}-c_{i}$. The possible outcomes of the market specify which pairs of buyer-seller end up making a transaction and at what price. One seeks a stable outcome, meaning that no pair consisting of a buyer and a seller can make an arrangement that is more satisfactory to both than the given one. Shapley and Shubik (1971) show that a stable outcome is supported by an equilibrium price, and furthermore, that there is a minimum equilibrium price vector and a maximal one. The strategic properties are the following ones:

(a) each buyer reaches his incremental value, or Vickrey payment, at a stable arrangement supported by the minimum equilibrium price vector. Hence, selecting the minimum equilibrium price vector gives the incentives to all buyers to reveal their true valuations. ${ }^{3}$ Similar results hold for the sellers by selecting the maximal price.

(b) no pair of buyer-seller can both obtain more than their incremental values by misrepresenting their preferences (Demange 1987).

Our purpose is to explore the extent to which properties (a) and (b) hold true in a coalitional formation model that extends the assignment game. We retain two main features: not all coalitions can form and utility is transferable within a coalition.

\footnotetext{
${ }^{3}$ This property was shown by Leonard (1983) through linear programming and by Demange (1982) and Demange et al (1986) through an algorithm in an appropriate graph, algorithm which can be interpreted as a multi-item Vickrey auction. The existence of a stable outcome most favorable to buyers (or to sellers) and its incentive properties extend to a setup with non transferable utility (Demange and Gale 1985).
} 


\subsection{The coalitional formation model}

A finite set of players, the "society", $N=\{1, \ldots, n\}$, may organize themselves into pairwise disjoint coalitions, where as usual a coalition is a non empty subset of $N$.

A collection $\mathcal{C}$ describes the set of admissible coalitions : coalitions outside $\mathcal{C}$ cannot form. Throughout the paper, singletons are admissible. The restrictions on coalition formation may stem from organizational or institutional reasons as illustrated in section 2.2. Our main interest is to relate collections $\mathcal{C}$ and strategic properties such as stated in (a) and (b).

Players only care about the coalition they join and the amount of money they receive or give. Player $i$ 's preferences are represented by a utility function $u_{i}$ defined over the coalitions that include $i: u_{i}(S)$ gives in term of money the utility for $i$ to be a member of coalition $S$. One interpretation is that $i$ derives an "intrinsic" utility for being a member of $S$. Another interpretation is that, once formed, a coalition selects a given alternative independently of the preferences of its members. In that case, $u_{i}(S)$ represents $i$ 's indirect utility for the alternative chosen by $S$. Utility functions are not necessarily increasing when an additional person is added to a coalition: an individual may dislike this person, or may not enjoy large coalitions. Thus, I shall assume that any utility function is admissible. The set of admissible $i$ 's utility functions is denoted by $\mathcal{U}_{i}$. The $n$-tuple $u=\left(u_{i}\right)_{i \in N}$ is a preferences profile and $\mathcal{U}=\bigotimes_{i=1, ., n} \mathcal{U}_{i}$ the set of admissible profiles.

A coalition $S$ that forms can decide to implement transfers between its members, $\left(t_{i}\right)_{i \in S}$. Player $i$ receives $t_{i}$ (positive or negative), hence achieves a utility level or payoff of $u_{i}(S)+t_{i}$. Feasibility requires total transfers within the coalition to be non-positive: $\sum_{i \in S} t_{i} \leq 0$. Thus, if $S$ forms, any payoff $\left(x_{i}\right)_{i \in S}$ that satisfies $\sum_{i \in S} x_{i} \leq \sum_{i \in S} u_{i}(S)$ can be achieved by $S$ alone through adequate transfers. This leads us to define the value of $S$ by

$$
V_{u}(S)=\sum_{i \in S} u_{i}(S)
$$

The society may split into several self-sufficient groups owing to individuals' preferences - when players dislike large coalitions for instance- or because of the constraints on coalitions as specified by the set $\mathcal{C}$-as in an assignment game in which only small coalitions can form. A coalition structure, as defined by Aumann and Dreze (1974), describes how players organize themselves into coalitions that are pairwise disjoint (hence membership to a coalition is exclusive) and self-sufficient (which excludes transfers across coalitions). Since there are no spill-over effects across coalitions, the stability notion based on the absence of blocking extends to coalition structures.

The following definitions of structures, blocking, and core are adapted so as to account for the set of admissible coalitions.

$\mathcal{C}$-partition $A \mathcal{C}$-partition is a partition $\pi=\left(S_{\ell}\right)_{\ell=1, \ldots, L}$ made of elements in $\mathcal{C}: S_{\ell} \in \mathcal{C}$ for each $\ell=1, \ldots, L . \Pi_{\mathcal{C}}(S)$ denotes the set of all $\mathcal{C}$-partitions of coalition $S$.

$\mathcal{C}$-structure $A \mathcal{C}$-(coalition) structure of $N$ is given by $a=(\pi, t)$ where $\pi$ is a $\mathcal{C}$-partition of $N$ and $t=\left(t_{i}\right)_{i \in N}$ specifies transfers that are balanced within each element in $\pi: \sum_{i \in S_{\ell}} t_{i} \leq 0$ for each $S_{\ell}$ in $\pi$. The utility level reached by $i$, denoted by $\tilde{u}_{i}(a)$, is $\tilde{u}_{i}(a)=u_{i}\left(S_{\ell(i)}\right)+t_{i}$ where $S_{\ell}(i)$ is the unique 
coalition of which $i$ is a member.

The coalition structure $a$ is said to be blocked by $T$ if

$$
\sum_{i \in T} \tilde{u}_{i}(a)<V_{u}(T)
$$

The blocking condition is justified as usual. Recall that coalition $T$ can achieve to its members any payoff that sums to $V_{u}(T)$. Thus, if (2) is met, each individual in $T$ could be made better off than under structure $a$. The stability notion follows.

$\mathcal{C}$-stability and $\mathcal{C}$-core Given a profile $u$, a $\mathcal{C}$-stable structure $a=(\pi, t)$ is a $\mathcal{C}$-structure that is not blocked by any coalition in $\mathcal{C}$. The payoff vector $\left(\tilde{u}_{i}(a)\right)_{i=1, \ldots, n}$ of a $\mathcal{C}$-stable structure is called $\mathcal{C}$-stable. The set of $\mathcal{C}$-stable structures is called the $\mathcal{C}$-core.

The collections for which a stable structure exists for any profile are of particular interest.

Guarantee of stability $A$ collection $\mathcal{C}$ guarantees stability on the set of profiles $\mathcal{U}$ if for any $u$ in $\mathcal{U}$ the $\mathcal{C}$-core is non empty.

The guarantee of stability imposes quite severe restrictions. To illustrate why, assume that collection $\mathcal{C}$ contains a Condorcet triple, that is three coalitions $S_{i}, i=1,2,3$, which intersect each other but whose overall intersection is empty : $S_{i} \cap S_{j} \neq \emptyset$ and $S_{1} \cap S_{2} \cap S_{3}=\emptyset$. No $\mathcal{C}$-stable structure exists at a profile for which the value of each $S_{i}$ is equal to 1 and the value of other coalitions are nil. Thus, the absence of a Condorcet triple is necessary for a collection to guarantee stability. It is not sufficient. Kaneko and Wooders (1982) provide a necessary and sufficient condition in terms of the balanced families introduced by Shapley (1967). A family $\mathcal{B}$ of subsets of $N$ is said to be balanced if there are nonnegative weights $\left(\gamma_{S}\right)_{S \in \mathcal{B}}$ such that $\sum_{S, i \in S} \gamma_{S}=1$ for each $i$. A partition is a balanced family (take weights equal to 1 ).

Partition property $A$ collection $\mathcal{C}$ satisfies the partition property if any balanced family composed with coalitions in $\mathcal{C}$ contains a partition.

Thanks to Scarf theorem (1967), the partition property is sufficient for $\mathcal{C}$ to guarantee stability on any set of utility profiles. It is also necessary because the set $\mathcal{U}$ is rich enough to generate all super-additive games. As expected, the partition property excludes Condorcet triples: A Condorcet triple can be completed by singletons so as to obtain a balanced family that is not a partition.

\subsection{Illustrative Examples}

- 1 - The assignment game fits the framework by taking as admissible coalitions the singletons and the pairs of buyer-seller. Observe that the game represents any situation in which the society is divided into two types of agents who are constrained to interact through exclusive, bilateral contracts. Stability is guaranteed because the partition property holds. ${ }^{4}$ When instead any pair in the society

\footnotetext{
${ }^{4}$ Existence of a stable outcome was shown through linear programming by Shapley and Shubik (1971) in the assignment game and through the acceptance algorithm by Gale and Shapley (1962) in the marriage model (two-sided but without side payment). Thanks to the partition property, existence is ensured with non transferable utility (Quinzii 1984).
} 
is admissible, as in a roommate problem, there are Condorcet triples and stability is not guaranteed. Also, in the "bridge game" in which only coalitions with four individuals are admissible, stability is not guaranteed (Shubik 1971).

- 2 - The job market proposed by Crawford and Knoer (1981) generalizes the assignment game. Entities are still divided into two subgroups, say firms and workers, with firms possibly interested in hiring several workers (or buyers and sellers with buyers who may buy several objects). Here, apart from singletons, a coalition is admissible if it contains a single firm. With at least two firms and three workers, stability is not guaranteed : there are Condorcet triples such as $\left\{f_{1}, w_{1}, w_{2}\right\}$ $\left\{f_{2}, w_{2}, w_{3}\right\},\left\{f_{1}, w_{3}, w_{1}\right\}$. Some conditions on preferences are needed to ensure the existence of a stable structure. If for example the firm's preferences satisfy the gross substitutes assumption of Kelso and Crawford (1982) the core is non empty (this assumption differs from that of substitute players, see footnote 11). Interpreting entities as buyers and sellers, the package assignment model is obtained in which buyers are possibly interested in several objects (Bikhchandani and Ostroy 2002).

- 3 - Networks games. Individuals are linked through a network and only the connected coalitions can form (see Kalai et al. 1979 for an earlier analysis of stability in an exchange economy.) When the network is a simple path, a consecutive game is obtained in which individuals are ordered and admissible coalitions are intervals as in Greenberg and Weber (1993). More generally, when the network is a tree, individuals are partially ordered as in a hierarchical structure (Demange 2004). Stability is guaranteed for a tree (or disjoint trees), and fails when the network contains a cycle since a Condorcet triple of connected coalitions exists.

Before introducing our last example, it is worth noting some simple properties about sub-collections of a collection $\mathcal{C}$. First, if $\mathcal{C}$ satisfies the partition property, all its sub-collections satisfy it: their cores are non empty. Second, dropping some coalitions from a collection $\mathcal{C}$ has two effects. On one hand less coalitions can block. On the other hand less coalitions can form, hence less structures are feasible. As a result, the cores associated with nested collections cannot be compared. This remark applies to the collection of connected sets in a tree : dropping some connected sets generates truly different (non empty) cores. This is illustrated in the last example and in Section 5.

- 4 - Collection of single-lapping coalitions ${ }^{5}$ (Papai 2004). In the absence of transfers the core associated with single-lapping coalitions is single valued and strategy-proof. Such result does not extend to the side payments case. To see this, suppose $N$ to be admissible for instance. The single lapping property requires that only singletons are also admissible. Hence only two structures are feasible: $N$ or the partition of singletons. Given a profile, the unique stable structure is $N$ if it Pareto dominates the partition of singletons, and is the partition otherwise. Allowing transfers dramatically enlarges the set of stable payoffs: it coincides with all individually rational payoffs summing to $V_{u}(N)$ when $N$ is worth forming, that is when $V_{u}(N)>\sum_{i} u_{i}(i)$.

More generally, in a game with side payments, a non empty core is typically multi-valued. Two

\footnotetext{
${ }^{5}$ The conditions on admissible coalitions are (1) no pair have more than one element in common, and (2) any cycle of intersecting coalitions have the same (single) element in common. Surely, there is a tree for which all admissible coalitions are connected.
} 
routes are open: to study the strategy-proofness of selections of the core or to extend strategy-proofness to correspondences. They will be investigated in Section 3 .

\subsection{Incremental values and $\mathcal{C}$-stable payoffs}

A coalitional game as described by $V_{u}$ and $\mathcal{C}$ may not be a transferable utility (TU) game owing to the restriction on transfers across coalitions. In order to define players' incremental values, which will play a crucial role, we consider an auxiliary TU super-additive game $\bar{V}_{u}$, called the super-additive cover. Allow each coalition $S$ to split up into elements of $\mathcal{C}$ and to implement transfers across these elements. By choosing to partition into $\pi, S$ can achieve to its members any payoff that sum to $\sum_{T \in \pi} V_{u}(T)$, the sum of the values of the elements in the partition. Hence define the value of $\pi$ by $V_{u}(\pi)=\sum_{T \in \pi} V_{u}(T)$. The value $\bar{V}_{u}(S)$ is obtained by a $\mathcal{C}$-partition of $S$ whose value is maximal:

$$
\bar{V}_{u}(S)=\max _{\pi \in \Pi_{\mathcal{C}}(S)} V_{u}(\pi) .
$$

A partition that achieves $\bar{V}_{u}(S)$ is called optimal for $S$ (typically it is unique). $\bar{V}_{u}$ is clearly a super-additive characteristic function.

Observe that, in order to reach all shares of $\bar{V}_{u}(S)$, coalition $S$ needs to implement transfers across the distinct elements of an optimal partition (if any). This is why the characteristic function $\bar{V}_{u}$ does not exactly describe the coalitional game under consideration.

Incremental values An important payment for a coalition, in particular for a player, is the incremental value to the set of all remaining players, that is the incremental contribution that the coalition adds to total value. The incremental value of coalition $T$ (to the set of all remaining players) is defined by

$$
\bar{V}_{u}(N)-\bar{V}_{u}(N-T)
$$

The incremental value of $T$ gives an upper bound on the sum of the payoffs that players in $T$ can achieve at a stable payoff: if $T$ gets more, the sum of the payoffs to $N-T$ is strictly less than $\bar{V}_{u}(N-T)$ by feasibility, hence an admissible subset of $N-T$ blocks.

More generally, the incremental value of a coalition $T$ to a coalition $S$ disjoint of $T$ is defined as the difference $\bar{V}_{u}(S \cup T)-\bar{V}_{u}(S)$.

$\mathcal{C}$-stable payoffs As shown by Kaneko and Wooders (1982) the set of $\mathcal{C}$-stable payoffs is described by the set of inequalities

$$
\begin{aligned}
& \sum_{i \in N} x_{i} \leq \bar{V}_{u}(N) \text { and } \\
& \sum_{i \in S} x_{i} \geq V_{u}(S), S \in \mathcal{C} .
\end{aligned}
$$

Inequality (4) states that the payoff vector $x$ can be achieved through a partition of $N$, possibly by implementing transfers across coalitions. Inequalities (5) are the no blocking conditions. Hence a $\mathcal{C}$-stable payoff surely satisfies (4) and (5). Conversely, it suffices to show that a payoff $x$ that satisfies (4) and (5) can be achieved by a partition without transfers across coalitions. Let $\pi$ be an optimal 
partition for $N$. Since any coalition $S$ in $\pi$ is admissible, $V_{u}(S) \leq \sum_{i \in S} x_{i}$ by (5). Summing over the elements in the partition $\pi$ and using the feasibility condition (4) give

$$
V_{u}(\pi)=\sum_{S / S \in \pi} V_{u}(S) \leq \sum_{S / S \in \pi} \sum_{i \in S} x_{i}=\sum_{i \in N} x_{i} \leq \bar{V}_{u}(N)
$$

By optimality of $\pi, V_{u}(\pi)$ is equal to $\bar{V}_{u}(N)$. Hence all inequalities $V_{u}(S) \leq \sum_{i \in S} x_{i}$ for $S$ in $\pi$ are binding : $x$ can be achieved by partition $\pi$ without cross-transfers. ${ }^{6}$

Thanks to the description of stable payoffs, the partition property is used as follows. The maximal payoff that a coalition $T$ can achieve at a $\mathcal{C}$-stable structure is the value of the linear program in which the sum of the payoffs to $T$ is maximized under the feasibility and no blocking conditions. Up to a transformation, the feasible set of the dual program is composed of the weight vectors supporting some balanced families. Following Shapley (1967), the extreme points of the feasible set correspond to minimal balanced families. This is how the partition property comes into play : minimal balanced families are partitions. For $T$ admissible, this will allow us to compute the value of the dual and to recover the incremental values.

To simplify notation, $\{$,$\} is dropped when there is no possible confusion. For example S-i$ denotes $S-\{i\}$. Also $S+i$ denotes the set $S \cup\{i\}$.

\section{Defining manipulability}

In this section, no restriction is made on the collection $\mathcal{C}$. The core, if non empty, is typically multi-valued. Therefore, when a player contemplates misrepresenting his preferences he compares two subsets of coalition structures. Various notions of manipulability are possible, depending on how preferences over coalition structures are extended over subsets. Before discussing several possibilities, it is worth noting that, whatever the collection $\mathcal{C}$, no selection of the $\mathcal{C}$-core is strategy proof for all players.

\subsection{Manipulability of selections}

Given a collection $\mathcal{C}$, let $\mathcal{S}(u)$ be the $\mathcal{C}$-core at profile $u$. Let $f$ be a function that assigns to each profile $u$ in $\mathcal{U}$ a $\mathcal{C}$-structure. Function $f$ is said to be a selection of $\mathcal{S}$ if it assigns a $\mathcal{C}$-stable structure at each profile for which it is possible :

$$
f(u) \in \mathcal{S}(u) \text { for each } u \text { such that } \mathcal{S}(u) \neq \emptyset \text {. }
$$

As usual, given a profile $u,\left(v_{i}, u_{N-i}\right)$ denotes the profile with functions $v_{i}$ for $i$ and $u_{j}$ for other individuals. The following definitions are standard.

\footnotetext{
${ }^{6}$ The set of $\mathcal{C}$-stable payoffs also coincides with the core of the game $\bar{V}_{u}$, which is described by (4) and the stronger no blocking conditions $\sum_{i \in S} x_{i} \geq \bar{V}_{u}(S)$, any $S$ : if the payoffs to $S$ are less than $\bar{V}_{u}(S)$, then at least one admissible $T$ subset of $S$ does not get $V_{u}(T)$ and blocks.
} 
Individual $i$ can manipulate $f$ at $u$ if for some $v_{i}$ in $\mathcal{U}_{i} \tilde{u}_{i}\left(f\left(v_{i}, u_{N-i}\right)\right\}>\tilde{u}_{i}(f(u))$. Function $f$ is strategy-proof for individual $i$ if $i$ cannot manipulate $f$ at any profile.

Proposition 1 Let $f$ be a selection of $\mathcal{S}$ and $u$ a profile with a non empty core $\mathcal{S}(u)$. If $i$ 's payoff at $f(u)$ is strictly smaller than his maximum stable payoff, then $i$ can manipulate $f$ at $u$. Consequently, if the $\mathcal{C}$-stable payoffs are not all equal, then at least one individual can manipulate $f$ at $u$.

The proof is in the appendix. It relies on the following property: when the maximum stable payoff of individual $i$ is strictly larger than the minimal one, $i$ is never single at a stable structure. This is surely the case if $i$ does not reach his maximum stable payoff $M_{i}$ at $f(u)$. Then, by increasing $i$ 's utility for standing alone to some value $v_{i}(i)$ larger than $\tilde{u}_{i}(f(u))$ but smaller than $M_{i}$, the stable structures are simply reduced to those in which $i$ 's true payoff is at least $v_{i}(i)$. Thus $i$ is surely better off at $f\left(v_{i}, u_{N-i}\right)$.

\subsection{Manipulability of the core}

Let us consider now the manipulation of the whole correspondence $\mathcal{S}$, starting with a single individual. When player $i$ contemplates announcing $v_{i}$ instead of his true preferences $u_{i}$ he compares the set $\mathcal{S}\left(v_{i}, u_{N-i}\right)$ with the set $\mathcal{S}(u)$. If $i$ evaluates each set on the basis of its preferred elements, the associated manipulability concept may be qualified as "optimistic". This is the concept I shall use. There are various arguments for this choice, not necessarily based on an optimistic behavior as explained below. Let us first discuss the alternative notion that obtains with individuals considering the worst element of a set.

Pessimistic individuals Pessimistic individual $i$ can manipulate $\mathcal{S}$ at $u$ with $\mathcal{S}(u) \neq \emptyset$ if for some $v_{i}$ in $\mathcal{U}_{i}$

$$
\min \left\{\tilde{u}_{i}(b), b \in \mathcal{S}\left(v_{i}, u_{N-i}\right)\right\}>\min \left\{\tilde{u}_{i}(a), a \in \mathcal{S}(u)\right\} .
$$

In other words, a pessimistic individual can manipulate if misrepresenting his preferences allows him to improve upon strictly the minimum utility level he achieves at a $\mathcal{C}$-stable structure.

This concept leads to an impossibility result in our setup: $\mathcal{S}$ is manipulable by at least one pessimistic individual at any profile for which the nonempty set $\mathcal{S}(u)$ contains distinct payoffs. Consider a selection of $\mathcal{S}$ that picks out a worst $\mathcal{C}$-stable structure for player $i$ at any profile with a non empty core. Let $u$ be a profile with $\mathcal{S}(u) \neq \emptyset$. If pessimistic $i$ cannot manipulate $S$ at $u$, then the selection is non manipulable by $i$ at this profile. It follows from Proposition 1 that $i$ 's worst and best $\mathcal{C}$-stable payoffs coincide. Thus, $\mathcal{S}$ is not manipulable by pessimistic individuals at $u$ only if all stable payoffs are equal.

Optimistic individuals Optimistic individual $i$ can manipulate $\mathcal{S}$ at $u$ with $\mathcal{S}(u) \neq \emptyset$ if for some $v_{i}$ in $\mathcal{U}_{i}$

$$
\max \left\{\tilde{u}_{i}(b), b \in \mathcal{S}\left(v_{i}, u_{N-i}\right)\right\}>\max \left\{\tilde{u}_{i}(a), a \in \mathcal{S}(u)\right\} .
$$

Thus, an optimistic individual can manipulate if misrepresenting his preferences allows him to get some structure $\mathcal{C}$-stable for the "false" profile that he strictly prefers to any $\mathcal{C}$-stable structure for $u$. 
This concept is worth studying. A main interest is that a process based on truthful or "myopic" blocking is justified only if optimistic individuals cannot manipulate. The argument does not rely on how individuals evaluate a set of structures, in particular does not assume individuals to be optimistic. To clarify this point, consider a process in which feasible structures are proposed and can be refused by any admissible coalition. Let individuals indeed believe in the "theory" that a coalition forms to refuse a proposal if and only if it can block the proposal (in the sense of (2)). Thus the $\mathcal{C}$-core describes the possible outcomes of the coalition formation process. ${ }^{7}$ Let $i$ be able to manipulate at $u$ and $b$ a preferred structure in $\mathcal{S}\left(v_{i}, u_{N-i}\right)$. Suppose that $b$ is proposed at some point in time. Note that surely $b$ is blocked at $u$ and that $i$ is a member of any blocking coalition (because otherwise $b$ would not be stable at $\left.\left(v_{i}, u_{N-i}\right)\right)$. Thus if $i$ participates in a blocking coalition, he anticipates that the process will settle at a final outcome in $\mathcal{S}(u)$. Instead, by not participating, $i$ anticipates that $b$ is not refused at all because $b$ is not blocked by coalitions of which he is not a member. Since he is better off under the latter alternative, he does not join any tentative refusal, contradicting the assumption of myopic blocking. Therefore the $\mathcal{C}$-core yields a consistent prediction of a myopic blocking process only if it is non manipulable by optimistic individuals.

Optimistic manipulability is also interesting because it is a rather weak requirement. As a result, the correspondences that are not manipulable by optimistic individuals, as we shall display in next section, are a fortiori not manipulable for more stringent notion. In particular optimistic manipulability is easier than "unambiguous" manipulability as introduced by Nehring (2000) : An individual can manipulate without ambiguity if he strictly prefers any structure obtained by misrepresenting his preferences to any one under truthful revelation. Unambiguous manipulation implies both pessimistic and optimistic manipulation.

Example 1 : A manipulable core. Is the core manipulable in the optimistic sense ? Given that the absence of blocking is a strong condition, the question is worth raising. Let us give an example of a manipulable core with four individuals. ${ }^{8}$ Consider player 1 in the following game:

$V_{u}(2,3)=V_{u}(2,4)=c, V_{u}(1,3,4)=d, V_{u}(1,2,3,4)=1$, and all other values are nil.

Assume $c \leq 1$ and $d \leq 1$ so that $\{1,2,3,4\}$ is the efficient structure. The incremental value of player 1 is $(1-c)$. It is an upper bound on 1's stable payoffs. The only possible stable payoff at which it can be achieved is $(1-c, c, 0,0):\{2,4\}$ must receive $c$ (otherwise it blocks), which leaves 0 to player 3 ; similarly $\{2,3\}$ must receive $c$, which leaves 0 to player 4 . However payoff $(1-c, c, 0,0)$ is stable

\footnotetext{
${ }^{7}$ This raises the question of whether a decentralized process of myopic blocking eventually converges to a stable structure. For a well chosen sequence of blocking coalitions, convergence has been proved in specific contexts, by Roth and Vande Vate (1990) in two-sided matchings without money, by Diamantoudi et al. (2004) in the roommate problem, by Sengupta and Sengupta (1996) in TU games, and more recently, by Koczy and Lauwers (2001) for coalitional games with transfers as those in this paper. Note that with a finite set of outcomes, convergence of a specific sequence implies convergence under an assumption of random blocking.

${ }^{8}$ With three individuals, each individual reaches his incremental value at a stable payoff if the core is non empty, which ensures its non manipulability, as stated in next proposition 3. Normalize the game by setting $\bar{V}_{u}(N)=1$ and 0 to singletons. Let individual 1 get his incremental payoff $x_{1}=1-\bar{V}_{u}(2,3)$, which leaves exactly $\bar{V}_{u}(2,3)$ for $\{2,3\}$. The non blocking conditions $x_{3} \leq 1-\bar{V}_{u}(1,2)$ (for $\{1,2\}$ ) and $x_{2} \leq 1-\bar{V}_{u}(1,3)$ (for $\{1,3\}$ ) can be satisfied if $\bar{V}_{u}(2,3) \leq 2-\left(\bar{V}_{u}(1,2)+\bar{V}_{u}(1,3)\right)$, which holds under the non-emptiness of the core.
} 
only if $c+d \leq 1$ : For $c+d>1$, it is blocked by $\{1,3,4\}$ and 1 's maximum stable payoff is reached at the extreme point $(2-2 c-d, 1-d, c+d-1, c+d-1)$ in which each coalition $\{1,3,4\},\{2,3\}$, and $\{2,4\}$ exactly gets its value.

Assume $c+d>1$. By lowering his utility for $\{1,3,4\}$, player 1 decreases the value of coalition $(1,3,4)$ to some $d^{\prime}$. His maximal payoff is increased to $2-2 c-d^{\prime}$, or even to his incremental value $(1-c)$ if $c+d^{\prime} \leq 1$.

The definition of optimistic manipulability extends to coalitions as follows. Let $\left(v_{T}, u_{N-T}\right)$ denote the profile with functions $u_{i}$ for individuals not in $T$ and $v_{i}$ for those in $T$.

Coalition manipulability Optimistic $T$ can manipulate $\mathcal{S}$ at $u$ if there is $v_{T}$ and $b$ in $\mathcal{S}\left(v_{T}, u_{N-T}\right)$ for which

$$
\tilde{u}_{i}(b)>\tilde{u}_{i}(a), \forall a \in \mathcal{S}(u), \forall i \in T \text {. }
$$

Thus, the members of $T$ can manipulate if, by misrepresenting their preferences, a structure $b$ that they all prefer to each $C$-stable structure becomes stable. As for an individual, the members of a manipulating coalition are essential for blocking the preferred structure, as stated in the following proposition.

Proposition 2 Assume that optimistic coalition $T$ can manipulate $\mathcal{S}$ at $u$ and prefers structure $b$ as given by (6). Then $b$ is blocked at $u$ and any coalition that blocks $b$ intersects $T$.

Proof. The proof is straightforward. Let $b$ be blocked by $S$. If $S$ does not intersect $T$, then $S$ also blocks $b$ at $\left(v_{T}, u_{N-T}\right)$. But then $b$ cannot be $\mathcal{C}$-stable for $\left(v_{T}, u_{N-T}\right)$.

Arguing as for a single individual, it follows from Proposition 2 that a process of myopic blocking is justified only if no coalition $T$ can manipulate the core in the optimistic sense. Otherwise, assuming other coalitions to block truthfully, members of $T$ facing preferred structure $b$ can prevent $b$ to be refused and are surely better off in doing so.

\subsection{Incremental values and non manipulability}

The link between strategy-proofness and incremental values is known since the work of Vickrey (1961). The argument extends to correspondences and coalitions under optimistic manipulability.

Proposition 3 A coalition that achieves its incremental value at a stable structure for profile u cannot optimistically manipulate $\mathcal{S}$ at that profile.

Proof. Let coalition $T$ achieve its incremental value at a stable payoff for $u$. Assume by contradiction that $T$ can optimistically manipulate at $u$. Surely the payoffs to $T$ at the preferred structure $b$ (under the "true" preferences $u$ ) are larger than its incremental value. Thus, denoting by $x=\tilde{u}(b)$ the payoff vector, one has $\sum_{i \in T} x_{i}>\bar{V}_{u}(N)-\bar{V}_{u}(N-T)$, and by feasibility of $x, \sum_{i \in N} x_{i} \leq \bar{V}_{u}(N)$. These inequalities imply $\sum_{i \in N-T} x_{i}<\bar{V}_{u}(N-T)$, in contradiction with the stability of $b$ at profile $\left(v_{T}, u_{N-T}\right)$. 


\section{On the non manipulability of the set of stable structures}

From now on, the paper considers collections $\mathcal{C}$ that satisfy the partition property. Hence, the non emptiness of the set $\mathcal{S}(u)$ is guaranteed. Our first main result relies on Proposition 3.

Theorem 1 Let collection $\mathcal{C}$ satisfy the partition property. Then, at each profile, for each coalition $T$ in $\mathcal{C}$ there is a $\mathcal{C}$-stable structure at which that coalition reaches its incremental value. Therefore, no admissible coalition can optimistically manipulate the $\mathcal{C}$-core.

Applying the proposition to singletons gives the following corollary.

Corollary Let collection $\mathcal{C}$ satisfy the partition property. Then, for each individual, there is a most favorable $\mathcal{C}$-stable structure at which that individual reaches his incremental value. Therefore, no optimistic individual can manipulate the $\mathcal{C}$-core.

Note that without restrictions on coalitions, the maximum level that a player can achieve at a stable payoff may be strictly smaller than his incremental value, as in example 1.

Proof of Theorem 1. Index $u$ is dropped. The maximum that members of coalition $T$ can reach at a $\mathcal{C}$-stable structure is the maximum of $\sum_{i \in T} x_{i}$ over the set of $\mathcal{C}$-stable payoffs. Recall that this set is described by the feasibility constraint (4) and the no-blocking constraints (5). Furthermore, it is non empty under the partition property. Hence, the maximum payoff to $T$ at a $\mathcal{C}$-stable structure solves the linear program :

$\max _{x} \sum_{i \in T} x_{i}$ under $\sum_{i \in N} x_{i} \leq \bar{V}(N)(4)$ and $\sum_{i \in S} x_{i} \geq V(S), S \in \mathcal{C}(5)$

Furthermore the value of the program is finite, reached, and equals the value of the dual by standard linear programming results (see e.g. Gale 1960). Denote by $\lambda$ and by $\delta_{S}$ the multipliers associated respectively to (4) and the no-blocking condition (5) for coalition $S$. The dual ${ }^{9}$ writes as

$$
\begin{gathered}
\min _{\lambda, \delta \geq 0} \lambda \bar{V}(N)-\sum_{S} \delta_{S} V(S) \text { subject to } \\
\left\{\begin{array}{l}
\delta_{S} \geq 0 \text { for } S \in \mathcal{C}, \text { and } \delta_{S}=0 \text { for } S \notin \mathcal{C} \\
1+\sum_{S, i \in S} \delta_{S}=\lambda, i \in T \\
\sum_{S, i \in S} \delta_{S}=\lambda, i \notin T .
\end{array}\right.
\end{gathered}
$$

We shall use the following lemma proved in the appendix.

Lemma 1 Given a balanced family $\mathcal{B}$ included in $\mathcal{C}$ with weights vector $\left(\gamma_{S}\right)$, there is $\mu=\left(\mu_{\pi}, \pi \in\right.$ $\left.\Pi_{\mathcal{C}}(N)\right)$ a vector of weights on $\mathcal{C}$-partitions that satisfies:

$$
\mu_{\pi} \geq 0, \sum_{\pi} \mu_{\pi}=1 \text { and } \sum_{\pi, S \in \pi} \mu_{\pi}=\gamma_{S} \forall S \in \mathcal{B} .
$$

\footnotetext{
${ }^{9}$ Write the Lagrangean $\sum_{i \in T} x_{i}+\lambda\left(\bar{V}(N)-\sum_{i \in N} x_{i}\right)+\sum_{S \in \mathcal{C}} \delta_{S}\left(\sum_{i \in S} x_{i}-V(S)\right)$, as$$
\sum_{i \in T} x_{i}\left(1-\lambda+\sum_{S, i \in S} \delta_{S}\right)+\sum_{i \notin T} x_{i}\left(-\lambda+\sum_{S, i \in S} \delta_{S}\right)+\lambda \bar{V}(N)-\sum_{S \in \mathcal{C}} \delta_{S} V(S),
$$ 
Lemma 1 allows us to rewrite the dual program in terms of the vector $\mu$ in a form that is straightforward to solve. Let $(\lambda, \delta)$ satisfy (8). Consider the family of coalitions formed with $T$ and the $S$ that have a positive weight $\delta_{S}$. All these coalitions belong to $\mathcal{C}$ because $T$ is admissible by assumption. Also the family is balanced: the vector $\gamma$ identical to $\delta$ except for $T$ where $\gamma_{T}=\delta_{T}+1$ satisfies $\sum_{S, i \in S} \gamma_{S}=\lambda$ for each $i$ with $\lambda>0$ from (8). Thus, applying Lemma 1 to $\gamma / \lambda$ there is a non negative vector $\mu$ that satisfies:

$$
\sum \mu_{\pi}=\lambda, \sum_{\pi, T \in \pi} \mu_{\pi}=\delta_{T}+1 \text { and } \sum_{\pi, S \in \pi} \mu_{\pi}=\delta_{S}, \forall S \neq T .
$$

Furthermore, thanks to the relationships (10):

$$
\sum_{S} \delta_{S} V(S)+V(T)=\sum_{\pi} \mu_{\pi} V(\pi)
$$

Conversely, starting from any nonnegative $\mu=\left(\mu_{\pi}, \pi \in \Pi_{\mathcal{C}}(N)\right)$ that satisfies $\sum_{\pi, T \in \pi} \mu_{\pi} \geq 1$, one can associate $\lambda$ and a nonnegative vector $\delta$ by (10) so as to satisfy the constraints (8) of the dual. This allows us to write the dual in the variable $\mu=\left(\mu_{\pi}, \pi \in \Pi_{\mathcal{C}}(N)\right)$ as:

$$
\left\{\begin{array}{l}
\min _{\mu} \sum_{\pi \in \pi_{\mathcal{C}}(N)} \mu_{\pi}[\bar{V}(N)-V(\pi)]+V(T) \\
\mu_{\pi} \geq 0, \sum_{\pi, T \in \pi} \mu_{\pi} \geq 1 .
\end{array}\right.
$$

Since by definition $\bar{V}(N) \geq V(\pi)$, the minimum is reached by choosing $\mu_{\pi}=0$ for any $\pi$ that does not contain $T$. For a partition $\pi$ that contains $T, V(\pi) \leq \bar{V}(T)+\bar{V}(N-T)$. Thus the minimum is reached by choosing $\mu_{\pi}=1$ for $\pi$ that contains $T$ and an optimal partition of $N-T$. This gives that the value of the dual is $\bar{V}(N)-\bar{V}(N-T)$.

Can a non admissible coalition manipulate? In the assignment game, the answer is positive for a coalition of buyers (or of sellers) that can implement side payments. ${ }^{10}$ Let be one seller and two identical buyers. Buyers value the object at $s+1$, where $s$ is the seller's value for the object. At any stable outcome, buyers' payoffs are null. Both are made better off by announcing each one a valuation for the object equal to $s$ and deciding on a money transfer from the winner of the object to the loser. In network games, a non admissible coalition can make all its members strictly better off even without implementing transfers across its members, as illustrated now.

Example 2. Manipulation by non admissible coalitions. There are three individuals on a line with 1 in between and $\mathcal{C}$ is the collection of all connected sets (thus only $\{2,3\}$ is not admissible). Function $V_{u}$ is $V_{u}(1,2,3)=1, V_{u}(i)=0$, and $V_{u}(1, i)=c_{i}, i=2,3$, where $c_{i}$, is between 0 and 1 . The incremental values are $1,1-c_{3}$, and $1-c_{2}$ respectively for players 1,2 , and 3 .

\footnotetext{
${ }^{10}$ In Demange (1987), I gave a sufficient condition for the core not to be manipulable by any coalition, and showed it holds in assignment games. The condition requires the set of stable payoffs to satisfy a weak version of Von Neumann and Morgenstern external stability (1944) : For any payoff $y$ that is blocked, there is a coalition $T$ blocking $y$ that can be made at least as well off at a stable payoff $x^{\prime}: y_{i} \leq x_{i}^{\prime}$ for any $i$ in $T$ (but $T$ does not necessarily block $y$ via $x^{\prime}$ ). Under this condition, a coalition, whether admissible or not, cannot manipulate if it is unable to implement transfers among its members. The condition is not necessarily satisfied in our coalitional games, as shown by example 2 .
} 
Assume $c_{2}+c_{3}>1$ and consider players 2 and 3. Each one achieves his incremental value at the same (extreme) stable payoff $\left(c_{2}+c_{3}-1,1-c_{3}, 1-c_{2}\right\}$. As for the non admissible coalition $\{2,3\}$, its incremental value is equal to 1 . It is not reached at a stable payoff : subtracting the no-blocking conditions, $x_{1}+x_{i} \geq c_{i}, i=2,3$ to the feasibility constraint $2\left(x_{1}+x_{2}+x_{3}\right) \leq 2$ gives $x_{2}+x_{3} \leq 2-\left(c_{2}+c_{3}\right)<1$. Players 2 and 3 can be better off by falsifying their preferences as follows: 2 announces a lower utility for $\{1,2\}$, thereby lowering the value of $\{1,2\}$ hence increasing the incremental payoff of player 3 , and similarly 3 makes 2 better off by lowering her utility for $\{1,3\}$.

\section{$5 \quad$ Strategy-proof selections}

A selection of the core may be strategy-proof for a given subset of players, although not for all. For instance, under the partition property, selecting a core structure at which a given player achieves his incremental value is strategy-proof for that player (Theorem 1). More interesting, in an assignment game, selecting the minimum equilibrium price gives to all buyers the incentives to tell the truth because they simultaneously reach their incremental values. This section provides conditions on the collection $\mathcal{C}$ under which a selection of the $\mathcal{C}$-core is strategy-proof for a given subset of players. As a preliminary, next proposition states that such a selection must give to each of these players his incremental value.

Proposition 4 Let collection $\mathcal{C}$ satisfy the partition property. Given a coalition $T$ (not necessarily admissible) a selection of $\mathcal{S}$ is strategy-proof for each player in $T$ if and only if each one reaches his incremental value at any profile.

Proof. The if part follows from the standard argument. Conversely, a selection is strategy-proof for each player $i$ in $T$ only if each one achieves his maximal stable payoff, by Proposition 1 . Under the partition property, this maximal payoff is $i$ 's incremental value by the Corollary of Theorem 1 .

Given a profile $u$, whether players indeed reach simultaneously their incremental values at a stable payoff is characterized in terms of the value $\bar{V}_{u}$ in the following lemma.

Lemma 2 Let collection $\mathcal{C}$ satisfy the partition property. Given profile u, players in $T$ simultaneously reach their incremental values at a stable outcome if and only if

$$
\sum_{\alpha \in S}\left(\bar{V}_{u}(N)-\bar{V}_{u}(N-\alpha)\right) \leq \bar{V}_{u}(N)-\bar{V}_{u}(N-S) \text { for any } S \text { subset of } T .
$$

Of course, condition (12) is always satisfied for $S$ singleton. That the condition is necessary for $S$ non singleton is easy to understand because the incremental value of $S$, the right hand side, is an upper bound on the payoffs to $S$ at a stable structure. The converse is proved in the appendix. Note that if $S$ is admissible, the reverse inequality of (12) surely holds: since the incremental value of $S$ is reached at a stable structure it cannot be larger than the sum of the maximum each player gets, which is the sum of the incremental values under the partition property. This suggests that no strategy-proof selection exists for an admissible and non singleton $T$, as will be confirmed by our next results. 
In view of obtaining a core selection that is strategy-proof for $T$, our objective is to characterize the collections $\mathcal{C}$ for which conditions (12) are satisfied at all profiles.

\subsection{Substitute players and chains}

This section considers two players, $\alpha$ and $\beta$. Condition (12) applies only to $S=T=\{\alpha, \beta\}$, and can be rewriten as

$$
\bar{V}_{u}(N)-\bar{V}_{u}(N-\alpha) \leq \bar{V}_{u}(N-\beta)-\bar{V}_{u}(N-\alpha \beta)
$$

where to simplify $N-\alpha \beta$ denotes the set $N-\{\alpha, \beta\}$. Inequality (13) says that the incremental value of player $\alpha$ to $N-\alpha \beta$, the right hand side, is negatively affected by the arrival of player $\beta$ joining $N-\alpha \beta$, the left hand side. This is a weaker version of the substitutes property defined in Shapley and Shubik (1971).

Substitutes Two players are substitutes ${ }^{11}$ at $u$ if

$$
\bar{V}_{u}(S+\alpha \beta)-\bar{V}_{u}(S+\beta) \leq \bar{V}_{u}(S+\alpha)-\bar{V}_{u}(S) \text { all } S, \alpha \notin S, \beta \notin S
$$

Players are substitutes if the incremental value of one of the players to a coalition is not positively affected by the arrival of the other player in the coalition. Although a priori weaker, requiring condition (13) to be satisfied at any profile implies that the two players are substitutes at any profile. To see this, given profile $u$ and coalition $S$, change the values of $u$ into 0 for all coalitions that are not included in $S+\alpha \beta$. Then (13) applied to this new profile gives that (14) holds for $u$ and coalition $S$.

Collections for which two players are substitutes at any profile will be stated in terms of chains that we introduce now.

Chain $A$ chain between $\alpha$ and $\beta$ is defined by two families of admissible coalitions, $\left(S_{k}, k=1, . ., \ell+1\right)$ and $\left(T_{k}, k=1, . ., \ell\right)$ with $\ell \geq 0$, each formed with disjoint elements, that satisfy

$\alpha$ belongs to $S_{1}$ and $\beta$ to $S_{\ell+1}$, no $T_{k}$ contains $\alpha$ or $\beta$

$T_{k}$ intersects $S_{k}$ and $S_{k+1}, k=1, . ., \ell$.

For $\ell=0$, a chain is simply an admissible coalition that contains both $\alpha$ and $\beta$. For $\ell=1$, a chain is given by two disjoint coalitions, $S_{1}$ and $S_{2}$, one that contains $\alpha$, the other $\beta$, and a third coalition $T_{1}$ that intersects both $S_{1}$ and $S_{2}$ but contains neither $\alpha$ nor $\beta$. Figure 1 represents a chain of length 2 .

\footnotetext{
${ }^{11}$ In the package assignment model with one seller, Bikhchandani and Ostroy (2002) relate the substitutes property with the possibility for the Vickrey payoffs to be reached at a price equilibrium. Note that the gross substitutes condition introduced by Kelso and Crawford (1982) in a job market model (section 2.2) differs. It bears on how preferences of a firm $i$ over sets of hired workers vary with this set, that is, keeping our notation, on how $u_{i}(S+i)$ varies as workers $\alpha$ or $\beta$ (distinct from $i$ ) are added to the set of $S$ of hired workers.
} 


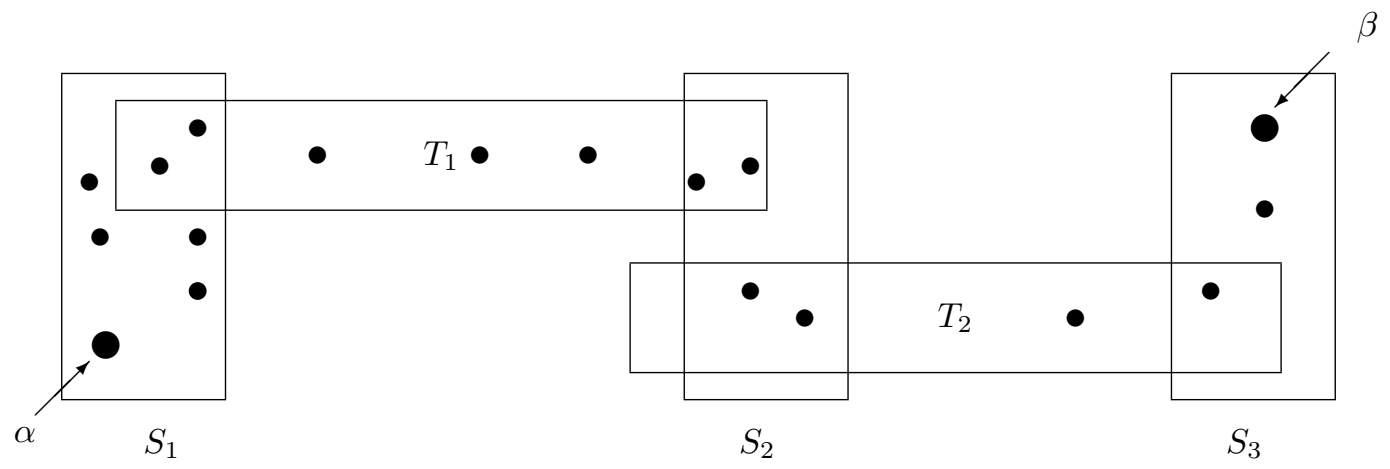

Figure 1

It is easy to understand that two players who are linked through a chain are not always substitutes. With a chain of length 2 for instance consider the game $V_{u}$ that assigns 1 to each element in the chain $S_{1}, S_{2}, S_{3}, T_{1}$ and $T_{2}$, and 0 otherwise. One has $\bar{V}_{u}(N)=3, \bar{V}_{u}(N-\alpha \beta)=\bar{V}_{u}(N-\alpha)=\bar{V}_{u}(N-\beta)=$ 2 , hence (13) does not hold. The incremental value of each single player is 1, equal to the incremental value of coalition $\{\alpha, \beta\}$ : each player cannot reach his incremental value at the same stable payoff. The argument extends to a chain of arbitrary length. ${ }^{12}$

To summarize, we have characterized the existence of a core selection strategy-proof for two players in terms of the substitute property or of condition (13) to be satisfied at any profile. Furthermore these properties imply the absence of a chain between the two players. The important new point in next theorem is the converse implication.

Theorem 2 Let collection $\mathcal{C}$ satisfy the partition property. Consider two players $\alpha$ and $\beta$. The following properties are equivalent:

1. there is a selection of $\mathcal{S}$ that is strategy-proof for $\alpha$ and $\beta$

2. condition (13) is met for $\alpha$ and $\beta$ at any profile

3. players $\alpha$ and $\beta$ are substitutes at any profile

4. there is no chain between $\alpha$ and $\beta$.

Before giving the proof, let us illustrate the chain condition in two examples. In the first example, an assignment game, it is already known that two players on the same side are substitutes and we simply check that there is no chain between them. The second example is new and describes the absence of a chain between two players in a tree game (-3- in Section 2.2). Observe first that no coalition in a chain is a singleton (because $T_{k}$ intersects two disjoint sets, and similarly for $S_{k}$, $k=2, . ., \ell ; S_{1}$ intersects $T_{1}$ at a different player than $\alpha$, similarly for $\left.S_{\ell}+1\right)$.

In an assignment game, consider two sellers for instance, $s$ and $s^{\prime}$. No coalition contains both sellers, thus there is no chain of length 0 . For a chain of positive length, it must be that $S_{1} \cap T_{1}$ is a buyer $b_{1}$, since seller $s$ does not belong to $T_{1}$. Hence $T_{1} \cap S_{2}$ is a seller $s_{1}$ (because otherwise it would be $b_{1}$, who would belong to the disjoint sets $S_{1}$ and $S_{2}$ ). Thus $S_{2} \cap T_{2}$ is a buyer (because otherwise

\footnotetext{
${ }^{12}$ Given a chain of length $\ell$, let $V_{u}$ assign to each element $S_{k}$ or $T_{k}$ in the chain, and 0 otherwise. Then $\bar{V}_{u}(N)=\ell+1$, $\bar{V}_{u}(N-\alpha \beta)=\bar{V}_{u}(N-\alpha)=\bar{V}_{u}(N-\beta)=\ell$, hence $(13)$ does not hold at $S=N$.
} 
it would be $s_{1}$, who would belong to both $T_{1}$ and $T_{2}$ ). Continuing this way, $T_{\ell} \cap S_{\ell+1}$ is a seller, who can only be $s^{\prime}$. But then $T_{\ell}$ contains $s^{\prime}$, in contradiction with a chain.

Example 3. Substitute players in a tree. In a tree game let two players $\alpha$ and $\beta$ be linked through player 1 as in Figure 2. Players $\alpha$ and $\beta$ are substitutes if $\mathcal{C}$ is the set of all connected coalitions except those that contain both $\alpha$ and $\beta$.

Before showing this, let us interpret the collection. Let $N_{\alpha}$ the set of individuals who are connected to 1 through $\alpha$ : they are in the subtree $G_{\alpha}$ containing $\alpha$ but not 1 (see Figure 2) and similarly $N_{\beta}$ for $\beta$. In a feasible partition, if a coalition is formed with 1 and one of the players, say $\beta$, then $N_{\alpha}$ is "left" alone and forms a partition. Thus, players $\alpha$ and $\beta$ can be interpreted as substitutes vis a vis player 1 who chooses which subtree is left alone.

By contradiction, consider a chain between $\alpha$ and $\beta$. Since no admissible coalition contains both $\alpha$ and $\beta$, the chain is of positive length $\ell$. We show that both $S_{1}$ and $S_{\ell+1}$ contains 1 , in contradiction with the fact that they must be disjoint in a chain of positive length. Suppose that $S_{1}$ does not contain 1: $S_{1}$ is a subset of $N_{\alpha}$. Then by definition $T_{1}$ intersects $N_{\alpha}$ (since it intersects $S_{1}$ ), is connected and does not contain $\alpha$ : it is included in $N_{\alpha}$. The same argument inductively shows that all sets in the chain are included in $N_{\alpha}$, hence do not contain 1 . Thus no set in the chain contains $\beta$, a contradiction: $S_{1}$ contains 1. That $S_{\ell+1}$ contains 1 follows from a similar argument.

In general, two players are connected through a path, say $(\alpha, 1, . ., m, \beta)$. We leave the reader to check that $\alpha$ and $\beta$ are substitutes under an additional condition: In the subtree obtained by deleting $G_{\alpha}$ and $G_{\beta}$ there must be no chain between 1 and $m$.

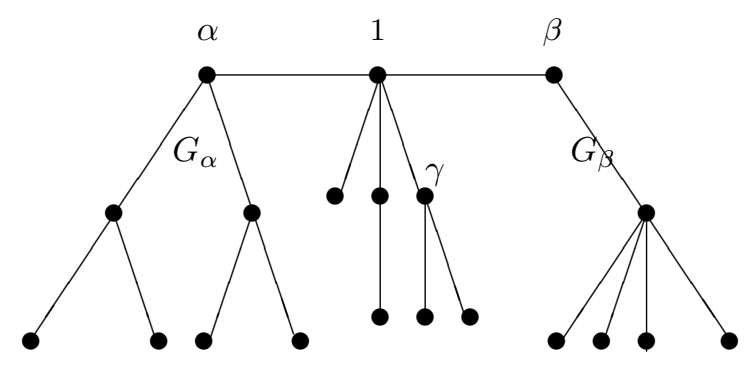

Figure 2

Proof of Theorem 2. That 1 is equivalent to 2 follows from Proposition 4 and Lemma 2, and that $2 \Rightarrow 3 \Rightarrow 4$ has been shown in the text.

It remains to prove that 4 implies 2. Let $\pi$ be a partition for $N$ and $\pi^{*}$ be a partition for $N-\alpha \beta$. We prove that, in the absence of a chain between $\alpha$ and $\beta$, all the elements of $\pi$ and $\pi^{*}$ can be assigned so as to get two partitions, one of $N-\alpha$ and one of $N-\beta$. This implies that (13) holds for any value $V_{u}$ on $\mathcal{C}$ by choosing for $\pi$ and $\pi^{*}$ an optimal partition for $N$ and $N-\alpha \beta$ respectively.

Starting from $\pi_{\alpha}=\pi_{\beta}=\emptyset$, the following algorithm assigns alternatively elements of $\pi$ and $\pi^{*}$ to $\pi_{\alpha}$ and $\pi_{\beta}$ so as to end up with partitions of $N-\beta$ and $N-\alpha$. Let $S_{\gamma}$ be the element of $\pi$ that 
contains $\gamma$ for $\gamma=\alpha, \beta$. $S_{\alpha}$ and $S_{\beta}$ are distinct since there is no admissible coalition containing both $\alpha$ and $\beta$ (no chain of length 0 ). Also if either one is a singleton the result is trivial. If $S_{\alpha}=\{\alpha\}$ for example, dropping $\{\alpha\}$ from $\pi$ and adding it to $\pi^{*}$ give two partitions of $N-\alpha$ and $N-\beta$ respectively.

Step 1

(i) assign $S_{\alpha}$ to $\pi_{\alpha}$ and $S_{\beta}$ to $\pi_{\beta}$.

(ii) assign all elements of $\pi^{*}$ that intersect $S_{\beta}$ to $\pi_{\alpha}$ (there are some since $S_{\beta}$ is not reduced to $\{\beta\}$ ), and those that intersect $S_{\alpha}$ to $\pi_{\beta}$.

Note that no element is assigned twice: otherwise there is $T$ in $\pi^{*}$ that intersects both $S_{\beta}$ and $S_{\alpha}$. Since $T$ does not contain $\alpha$ nor $\beta\left(\pi^{*}\right.$ is a partition of $\left.N-\alpha \beta\right)$, this gives a chain of length 1 , a contradiction.

Let $T_{\gamma}^{1}$ be the union of all $T$ assigned to $\gamma, \gamma=\alpha, \beta$. By construction,

$$
T_{\alpha}^{1} \supset S_{\beta}-\beta \text { and } T_{\beta}^{1} \supset S_{\alpha}-\alpha .
$$

If both inclusions are equalities, we are done. $\pi_{a}$ is a partition of $S_{\alpha} \cup S_{\beta}-\beta, \pi_{b}$ a partition of $S_{\alpha} \cup S_{\beta}-\alpha$ and the remaining (i.e. not yet assigned) elements of $\pi$ form a partition of $N-S_{\alpha} \cup S_{\beta}$ and similarly for $\pi^{*}$. Completing $\pi_{\alpha}$ with the remaining elements of $\pi$ and $\pi_{\beta}$ with the remaining elements of $\pi^{*}$ (or the converse) give two partitions, one of $N-\beta$ the other for $N-\alpha$. If not, go to step 2 .

\section{Step 2}

(i- $\alpha$ ) if $T_{\beta}^{1} \neq S_{\alpha}-\alpha$, assign to $\pi_{\alpha}$ any remaining $S$ of $\pi$ that intersects a $T$ assigned to $\pi_{\beta}$ in step 1. For such $S$, there is a sequence $\left(S_{\alpha}, T\right.$ in $\left.\pi_{\beta}, S\right)$ where $T$ intersects the adjacent sets.

(i- $\beta$ ) if $T_{\alpha}^{1} \neq S_{\beta}-\beta$, do similarly for $\beta$.

Since by assumption one inclusion in (15) is strict, surely (i- $\alpha$ ) or (i- $\beta$ ) holds, so that at least a new set is assigned. Furthermore no $S$ is assigned twice: otherwise, the two sequences $\left(S_{\alpha}, T\right.$ in $\pi_{\beta}$, $S)$ and $\left(S_{\beta}, T^{\prime}\right.$ in $\left.\pi_{\alpha}, S\right)$ would give $\left(S_{\alpha}, T, S, T^{\prime}, S_{\beta}\right)$, a chain of length $2\left(T\right.$ and $T^{\prime}$ are surely distinct).

Let $S_{\gamma}^{2}$ be the union of all $S$ assigned to $\pi_{\gamma}$ at step 1 or 2 for $\gamma=\alpha, \beta$. By construction

$$
S_{\alpha}^{2}-\alpha \supset T_{\beta}^{1} \text { and } S_{\beta}^{2}-\beta \supset T_{\alpha}^{1} .
$$

If all inclusions are equalities, we are done, arguing as above. If not, go to step (ii- $\alpha$ ) or (ii- $\beta$ ) or both.

(ii- $\alpha$ ) if $S_{\beta}^{2}-\beta \neq T_{\alpha}^{1}$ assign to $\pi_{\alpha}$ all the remaining elements of $\pi^{*}$ that intersect $S_{\beta}^{2}$ (those that intersect $S_{\beta}^{2}$ and that have been assigned at step 1 have been also assigned to $\pi_{\alpha}$ ). Thus for any such $T$ there is a sequence $\left(S_{\beta}, T_{1}\right.$ in $\pi_{\alpha}, S$ in $\left.\pi_{\beta}, T\right)$ in which the new assigned set $T$ intersects $S$ and is disjoint from $T_{1}$.

(ii- $\beta$ ) if $S_{\alpha}^{2}-\alpha \neq T_{\beta}^{1}$ do similarly for $\beta$.

Observe that at least a new set of $\pi$ or of $\pi^{*}$ is assigned since (ii- $\alpha$ ) or (ii- $\beta$ ) holds by assumption. Also, no new set $T$ is assigned twice: otherwise, the two sequences starting from $S_{\beta}$ and from $S_{\alpha}$ leading to $T$ would give a chain of order 3 .

Continuing this way, the process surely stops since at each step there is at least a new set of $\pi$ or of $\pi^{*}$ assigned. 


\subsection{Extension to more than two players}

A core selection that is strategy-proof for a subset $T$ is strategy-proof for each pair in the subset. Hence each pair of players in the subset must be substitutes. It turns out that it is sufficient.

Proposition 5 Let collection $\mathcal{C}$ satisfy the partition property and $T$ be a coalition. There is a selection of the $\mathcal{C}$-core that is strategy-proof for each member of $T$ if and only each pair of players in $T$ are substitutes at any profile, or equivalently if and only if there is no chain between any pair of players in $T$.

Observe that no subset of $T$ is in $\mathcal{C}$ because two players who belong to the same admissible coalition are not substitutes. To illustrate, consider a tree game (example 3 in the previous section). Let coalition $T$ be formed with players who are directly linked to the same player $1(\alpha, \beta$, and $\gamma$ for instance in Figure 2). Take $\mathcal{C}$ to be a collection of connected coalitions in which no one contains more than one member of $T$. Then every pair of players are substitutes.

Proof. From Theorem 2 applied to pairs we know that any two players in $T$ must be substitutes. To show the converse, assume each pair of players in $T$ be substitutes. From Lemma 2, we have to prove that for every non singleton $S$ included in $T$ inequality

$$
\sum_{\alpha \in S}\left(\bar{V}_{u}(N)-\bar{V}_{u}(N-\alpha)\right) \leq \bar{V}_{u}(N)-\bar{V}_{u}(N-S)
$$

holds. The proof is by induction on the cardinality of $T$. It is known to hold for $T$ of cardinality 2 (Theorem 2). Assuming it to be true for any coalition with strictly less than $p$ elements, consider $T$ with cardinality $p$. W.l.o.g. $T=\{1, \ldots, p\}$. By the induction assumption, (12) is satisfied for any $S$ strict subset of $T$. Let us prove it for $S=T$. Since $p$ and $i$ are substitutes for $i=1, . ., p-1$, the incremental value of $p$ over a set containing $i$ is not decreased when $i$ is subtracted from the set. In particular the incremental value of $p$ over $N-\{p\}$ is not larger than over $N-\{p-1, p\}$, which in turn is not larger than the incremental value of $p$ over $N-\{p-2, p-1, p\}$ and so on. Iterating these inequalities from $i=p-1$ down to to $i=1$ gives that the incremental value of $p$ over $N-\{p\}$ is not larger than over $N-\{1, \ldots, p\}$ :

$$
\bar{V}_{u}(N)-\bar{V}_{u}(N-\{p\}) \leq \bar{V}_{u}(N-\{1, . ., p-1\})-\bar{V}_{u}(N-\{1, \ldots, p\}) .
$$

The induction assumption applied to $\{1, \ldots, p-1\}$ gives

$$
\sum_{\alpha=1, . ., p-1}\left(\bar{V}_{u}(N)-\bar{V}_{u}(N-\alpha)\right) \leq \bar{V}_{u}(N)-\bar{V}_{u}(N-\{1, . ., p-1\})
$$

Adding the two above inequalities gives that (12) is satisfied for $T$, the desired result.

\section{Discussion and concluding remarks}

The core of an assignment game enjoys nice strategic properties. This paper has shown how these properties extend to some coalitional games. In particular, we have displayed conditions on the set 
of admissible coalitions under which it is justified to use the predictions of coalition formation given by the (properly extended) core. The incentive properties of social choice correspondences depend on how manipulability is defined, that is how preferences over alternatives are extended to preferences over sets of alternatives. As a result, the literature has obtained impossibility results that extend Gibbard-Satterthwaite theorem as well as a few possibility results. In the setting of this paper, the "optimistic" extension plays a crucial role. We have shown that in some sense it is this weaker notion that allows for possibility results. Furthermore, it helped us to understand better the incentive properties of selections of the core. In particular, the existence of a selection that is strategy-proof for a subset of players has been characterized in terms of the collection of admissible coalitions. Albeit limited, these results make more precise the links between the structure of collaboration and strategic behavior.

\section{References}

Aumann, Robert and Jacques Dreze (1974) "Cooperative Games with Coalition Structures," International Journal of Game Theory 3, 217-237.

Ausubel, Lawrence (2004) "An Efficient Ascending-Bid Auction for Multiple Objects" American Economic Review 94-5, 1452-1475.

Barbera Salvador and Anke Gerber (2003) "On coalition formation: Durable Coalition structures," Mathematical Social Sciences 45, 185-203.

Bikhchandani, Sushil and Joseph Ostroy (2002) "The Package Assignment Model," Journal of Economic Theory 107: 377-406.

Bikhchandani, Sushil and Joseph Ostroy (2006) "Ascending price Vickrey auctions" Games and Economic Behavior 55 215-241.

Chwe Michael Suk-Young (1994) "Farsighted Coalitional Stability," Journal of Economic Theory 63, 299-325.

Clarke, E. (1971) "Multipart Pricing of Public Goods." Public Choice 11, 17-33.

Cramton, Peter C, (1995). "Money Out of Thin Air: The Nationwide Narrowband PCS Auction," Journal of Economics and Management Strategy, Blackwell Publishing, vol. 4(2), pages 267-343, Summer.

Crawford Vincent and Elsie Marie Knoer (1981) "Job matching with heterogenous Firms and Workers," Econometrica 49, 437-450.

Demange, Gabrielle (1982) "Strategyproofness in the assignment Market Game," Working paper, Laboratoire d'Econométrie de l'Ecole Polytechnique.

Demange, Gabrielle and David Gale (1985) "The Strategy Structure of Two Sided Matching Markets", Econometrica 53 (4), 1985, 873-888.

Demange, Gabrielle, David Gale, and Marilda Sotomayor (1986) "Multi-Item Auctions" Journal of 
Political Economy 94 (4), 863-872.

Demange, Gabrielle (1987) "Nonmanipulable Cores," Econometrica 55, 1057-74.

Demange, Gabrielle (2004) "On group stability in hierarchies and networks," Journal of Political Economy 112 (4), 754-78.

Diamantoudi, Effrosyni, Miyagawa, Eiichi and Xue, Licun (2004) "Random paths to stability in the roommate problem," Games and Economic Behavior vol. 48(1), 18-28.

Diamantoudi, Effrosyni and Licun Xue (2003) "Farsighted stability in hedonic games," Social Choice and Welfare 21, 39-61.

Dubins, L.E. and D. Freedman (1981) "Machiavelli and the Gale-Shapley Algorithm," American Mathematical Monthly 88, 485-496.

Gale, David (1960): The Theory of Linear Economic Models, McGraw-Hill, New-York.

Gale, David and Lyod S Shapley (1962) "College admissions and the stability of marriage," American Mathematical Monthly vol. 69, 9-15.

Greenberg, Joseph and Shlomo Weber (1994) "Stable coalition structures in consecutive games," in Frontiers in Game Theory Binmore, K., Kirman, A. and P. Tani, eds., MIT Press, Cambridge.

Groves, T. (1973). "Incentives in Teams," Econometrica 41, 617-631.

Kalai, Ehud, Andrew Postlewaite and John Roberts (1978) "Barriers to Trade and Disadvantageous Middlemen: Nonmonotonicity of the Core," Journal of Economic Theory 19, 200-210.

Kaneko, Mamoru and Myrna Wooders (1982) "Cores of Partitioning Games," Mathematical Social Sciences 3 , 313-327.

Kelso, Alexander S., and Vincent P. Crawford (1982) "Job Matching, Coalition Formation, and Gross Substitutes," Econometrica 50 , 1483-1504.

Koczy, Laszlo and Luc Lauwers (2001) "The coalition structure core is accessible" mimeo.

Leonard, H. B. (1983) "Elicitation of Honest Preferences for the Assignment of Individuals to Positions." Journal of Political Economy 91, 461-479.

Nehring Klaus (2000) "Monotonicity implies strategy-proofness for correspondences," Social choice and Welfare 17, 367-375.

Ostroy Joseph and Louis with L. Makowski (1987) "Vickrey-Clarke-Groves Mechanisms and Perfect Competition," Journal of Economic Theory 42, 244-261.

Papai Szylvia (2004) "Unique Stability in Simple Coalition Formation Games," Games and Economic Behavior 48, 337-354.

Quinzii, Martine (1984) "Core and Competitive Equilibria with Indivisibilities," International Journal of Game Theory, 13, 41-60.

Roth, Alvin E. and Andrew Postlewaite (1977) "Weak versus Strong Domination in a Market with 
Indivisible Goods," Journal of Mathematical Economics 4, 131-137.

Roth, Alvin .E. and J.H. Vande Vate (1990) "Random paths to stability in two-sided matchings," Econometrica 59, 1475-80.

Scarf Herbert E. (1967) "The core of an n-person game," Econometrica 35, 50-69.

Sengupta A. and K. Sengupta (1996) "A property of the core," Games and Economic Behavior 12, 266-273.

Shapley, Lloyd S., (1967), "On Balanced Sets and Cores," Naval Research Logistics Quarterly 14, 453-460.

Shapley, Lloyd S. and Martin Shubik (1971) "The assignment game I: the core," Int. J. Game Theory $1,111-130$.

Shubik, Martin (1971) "The bridge game economy : An example of indivisibilities," Journal of Political Economy 909-912.

Sönmez, Tayfun (1999) "Strategy-Proofness and Essentially Single-Valued Cores," Econometrica 67: 677-689.

Vickrey, W. (1961) "Counter speculation, auctions, and competitive sealed tenders," Journal of Finance $16,8-37$.

Von Neumann, J. and and O. Morgenstern (1944) Theory of Games and Economic Behavior Princeton: Princeton University press, Second Ed., 1947.

\section{Appendix}

Proof of Proposition 1 Assume that $f(u)$ is a stable structure but not one preferred by $i$. Let $\left[m_{i}, M_{i}\right]$ be the non empty interval of $i$ 's payoffs at stable structures. Surely $m_{i} \leq \tilde{u}_{i}(f(u))<M_{i}$. To show that $i$ can manipulate, change $u_{i}$ into $v_{i}$ only by increasing the utility for standing alone, $u_{i}(i)$, to some value $v_{i}(i)$ larger than $u_{i}(f(u))$ and smaller than $M_{i}$. Denote $v=\left(v_{i}, u_{-i}\right)$. We show that $\mathcal{S}(v)$ is the subset of $\mathcal{S}(u)$ obtained by eliminating all the structures in which $i$ 's payoff is strictly less than $v_{i}(i)$. Thus $i$ is surely better off at $f(v)$ in $\mathcal{S}(v)$ by announcing $v_{i}$ : $i$ can manipulate.

Let $a=(\pi, t)$. Observe that the blocking conditions for $u$ and $v$ only differ at the singleton $\{i\}$. If the partition $\pi$ contains $\{i\}, a$ is stable for $v$ iff it is stable for $u$. If $\pi$ does not contain $\{i\}, a$ is stable for $v$ if and only if $\tilde{u}_{i}(a)=u_{i}\left(S_{\ell(i)}\right)+t_{i}$ is not less than $v_{i}(i)$.

It follows that if $i$ is never single in an optimal partition for $u$, at each $a$ in $\left.\mathcal{S}(v): \tilde{u}_{i}(a)\right) \geq$ $v_{i}(i)>\tilde{u}_{i}(f(u)): \mathcal{S}(v)$ is the subset of $\mathcal{S}(u)$ composed of the structures in which $i$ 's payoff is at least $v_{i}(i)$, as desired. It remains to show that the inequality $m_{i}<M_{i}$ implies that $i$ is never single at a stable structure. By contradiction, if $i$ is single, one has $m_{i}=u_{i}(i)$ (by feasibility) and $u_{i}(i)+\bar{V}_{u}(N-i)=\bar{V}_{u}(N)$ (by optimality of the partition). Hence $u_{i}(i)$ is exactly equal to $i$ 's incremental value, which is an upper bound of $i$ 's stable payoffs: this implies $M_{i} \leq u_{i}(i)=m_{i}$, the 
desired contradiction.

Proof of Lemma 1. Given a balanced family $\mathcal{B}$, let $\Delta$ be the set of its weight vectors. $\Delta$ is convex and compact, hence is the convex envelope of its extreme points. As shown by Shapley (1967), an extreme point of $\Delta$ is associated with a balanced family included in $\mathcal{B}$ that is minimal, meaning that it contains no other balanced family. Under the partition property, a minimal balanced family is a partition, Hence the extreme points of $\Delta$ are $\delta^{\pi}\left(\delta_{S}^{\pi}=1\right.$ for $S$ element of $\pi$ and 0 otherwise) where $\pi$ is a partition included in $\mathcal{B}$. Thus for any $\gamma$ in $\Delta$ there are non negative $\mu_{\pi}$ summing to 1 such that $\gamma_{S}=\sum_{\pi} \mu_{\pi} \delta_{S}^{\pi}$ for each $S$ in $\mathcal{B}$, which gives (9).

Proof of Lemma 2. Drop index $u$ in $V_{u}$. Consider the program of maximizing the sum of the payoffs to $T$ over the stable payoffs, as in the proof of Theorem 1. At a stable structure, a payoff to a player is at most equal to his incremental value. Therefore it suffices to show that under (12) the value of the program is the sum of the individuals' incremental values. Given a vector $(\lambda, \delta)$ that satisfies the constraints of the dual

$$
\left\{\begin{array}{l}
\delta_{S} \geq 0 \text { for } S \in \mathcal{C}, \text { and } \delta_{S}=0 \text { for } S \notin \mathcal{C} \\
1+\sum_{S, i \in S} \delta_{S}=\lambda, i \in T \text { and } \sum_{S, i \in S} \delta_{S}=\lambda, i \notin T .
\end{array}\right.
$$

Let $\mathcal{B}$ be the family of coalitions formed with all singletons $\{\alpha\}, \alpha$ in $T$, and the coalitions $S$ that have a positive weight $\delta_{S}$. $\mathcal{B}$ is made of elements of $\mathcal{C}$ and balanced. Using Lemma 1 , the dual is equivalent to

$$
\begin{gathered}
\operatorname{minimize} \sum_{\pi \in \Pi_{\mathcal{C}}} \mu_{\pi}[\bar{V}(N)-V(\pi)]+\sum_{\alpha \in S} V(\alpha) \\
\text { over } \mu \geq 0 \sum_{\pi,\{\alpha\} \in \pi} \mu_{\pi} \geq 1, \alpha \in T
\end{gathered}
$$

Since $\bar{V}(N) \geq V(\pi)$, the minimum is reached by choosing $\mu_{\pi}=0$ for any $\pi$ that contains no singleton $\{\alpha\}$ for $\alpha$ in $T$. Now let a partition $\pi$ with positive weight $\mu_{\pi}$ that contains several singletons $\{\alpha\}$ for $\alpha$ in a subset $S$ of $T, S$ of cardinality greater than 1 . Surely $V(\pi) \leq \bar{V}(N-S)+\sum_{\alpha \in S} V(\alpha)$. By (12) this implies that

$$
[\bar{V}(N)-V(\pi)] \geq \sum_{\alpha \in S}[\bar{V}(N)-\bar{V}(N-\alpha)-V(\alpha)]
$$

Note that $\bar{V}(N-\alpha)+V(\alpha)$ is the value of a partition $\pi^{\alpha}$ that contains $\{\alpha\}$ and an optimal partition for $N-\alpha$. Replace the partition $\pi$ by these partitions $\pi^{\alpha}$ for $\alpha$ in $S$ and assign to each the weight $\mu_{\pi}$ : the constraints (17) are still satisfied and the objective function can only decrease. It follows that the minimum of the dual is reached by partitions $\pi^{\alpha}, \alpha$ in $T$, each one with a weight equal to 1 . Therefore the value of the program is $\sum_{\alpha \in T}[\bar{V}(N)-\bar{V}(N-\alpha)]$, the sum of the individual incremental values. 